\title{
Se Niklas Luhman encontrasse Hakim Bey: o problema da comunicação
}

\author{
Marco Toledo de Assis Bastos' \\ ECA-USP \\ herrcafe@uol.com.br
}

\begin{abstract}
RESUMO: Esse artigo tenta um cruzamento entre a teoria dos sistemas sociais de Niklas Luhmann com as provocações do ensaísta e poeta Hakim Bey. O intuito é localizar um problema de natureza autenticamente comunicacional que só se torna visível no entrelaçar desses dois autores. De um lado, entendemos que Luhmann é um autor central para se pensar a comunicação, e de outro, pensamos que Bey pode oferecer mais que apenas um discurso de flagrante adesão ideológica. Sim, há uma dimensão epistemológica em Hakim Bey que pode ser cruzada com a teoria luhmanniana. Acima de tudo, assumimos total responsabilidade pela anomalia aqui apresentada.
\end{abstract}

PALAVRAS-CHAVE: Comunicação; Niklas Luhmann; Hakim Bey

\begin{abstract}
This article shall try working Niklas Luhmann's theory of social systems together with writer and poet Hakim Bey's ideas. The point is to go toward a genuine communication issue that will only get clear through a combination of these authors. On the one hand, we recognize Luhmann as a crucial author to think about communication. On the other hand, we acknowledge Bey may have more to offer than ordinary ideological orientations. Indeed there is in some extent an epistemological depth in Hakim Bey that might be worked together with Luhmann's theory. Above all, we take all responsibilities for this deviation presented at this time.
\end{abstract}

KEY WORDS: Communication; Niklas Luhmann; Hakim Bey

\footnotetext{
1 Marco Toledo de A. Bastos é mestre em Ciências da Comunicação, com ênfase em Teoria da Comunicação e Cibercultura. É pesquisador do Núcleo de Estudos Filosóficos da Comunicação (FiloCom) e Doutorando na Escola de Comunicações e Artes da Universidade de São Paulo (ECA-USP).
} 
RESUMEN: Este artículo intentará trabajar la teoría de Sistemas de Niklas Luhmann junto con las ideas del escritor y poeta Hakim Bey. El punto es situar una cuestión de la comunicación que solamente se logrará visible mediante la combinación de estos autores. Por un lado, creemos que Luhmann es un autor muy importante para se pensar la comunicación. Por otro lado, pensamos que Bey puede tener más a ofrecer que únicamente orientaciones ideológicas. Sí, hay en una dimensión epistemológica en Hakim Bey que se puede trabajar junto con la teoría de Luhmann. Sobre todo, asumimos todos los débitos por esta anomalía que en este momento se ha presentado.

PALABRAS CLAVES: Comunicación; Niklas Luhmann; Hakim Bey

RÉSUMÉ: Cet article essaie un croisement entre la théorie de systèmes sociales de Niklas Luhmann avec des provocations d'essaist et de poèt Hakim Bey. La intention est trouver une question de nature authentiquemente de communication qui deviendra seul évident par une combinaison de ces deux auteurs. D'un part nous identifions Luhmann comme un auteur très important pour penser la communication, et d'autre part nous entendons que Bey a plus à offrir que seulement un discours des orientations idéologiques ordinaires. Oui, il ya une dimension épistémologique dans Hakim Bey qui peut êtré croiser avec la théorie de Luhmann. Surtout, nous prendrons toutes les responsabilités de cette anomalie ici présentée.

MOTS-CLÉ: Communication; Niklas Luhmann; Hakim Bey.

A máquina de guerra nômade conquista sem ser notada e se move antes do mapa ser retificado

Hakim Bey ${ }^{2}$

Seres humanos não podem comunicar; Apenas a comunicação pode comunicar Niklas Luhmann ${ }^{3}$

\footnotetext{
2 Bey, Hakim. TAZ: Zona Autônoma Temporária. São Paulo: Conrad, 2001. (p.19). 3 „Der Mensch kann nicht kommunizieren; nur die Kommunikation kann kommunizieren“. Na versão inglesa: "Humans cannot communicate; only communication can communicate" Luhmann, Niklas. Wie ist Bewusstsein an Kommunikation beteiligt? Soziologische Aufklärung 6 - Die Soziologie und der Mensch. Opladen, 1995 (p.37).
} 
Esse artigo tentará um cruzamento de todo impossível: a teoria dos sistemas sociais de Niklas Luhmann com as provocações do ensaísta e poeta Hakim Bey. O intuito é localizar um problema de natureza autenticamente comunicacional que só se torna visível no entrelaçar desses dois autores. De um lado, entendemos que Luhmann é um autor central para se pensar a comunicação, e de outro, pensamos que Bey pode oferecer mais que apenas um discurso de flagrante adesão ideológica. Sim, há uma dimensão epistemológica em Hakim Bey que pode ser cruzada com a teoria luhmanniana. Acima de tudo, assumimos total responsabilidade pela anomalia aqui apresentada.

Hakim Bey (pseudônimo de Peter Lamborn Wilson) é um autor marcado pelo situacionismo sessentista 4 , movimento político e artístico que concebia intervenções no espaço público de maneira a romper com o establishment político e cultural. Os ensaios político-filosóficos de Bey produziram as concepções de anarquia ontológica e terrorismo poético e, por fim, de zona autônoma temporária, conceito derivado de seus estudos históricos sobre as utopias piratas. Uma TAZ (Temporary Autonomous Zone) pode ser uma porção de coisas e é vão defini-la com muita precisão tão logo.

O tema principal de Bey diz que o tecido social está prenhe de uma força libertadora, que por razões insuspeitas pode unir indivíduos temporariamente e se proliferar sucessivamente. Esses levantes temporários proporcionariam ao sistema social uma comunicação efetiva com os sistemas psíquicos. A proposição tem laivos de utilitarismo e de utopia social, no melhor estilo do libertarianismo norteamericano. Mas essa perspectiva política do anarquismo nova-iorquino é um veio interessante para acrescer às concepções de Niklas Luhmann e sua teoria da comunicação, que apresenta lacunas operacionais. Falta em Luhmann algum elemento que forneça mobilidade suplementar aos sistemas psíquicos nos

\footnotetext{
$4 \mathrm{O}$ situacionismo sessentista, grosso modo, tinha por meta questionar a natureza da arte, seu papel social, estético, político e a possibilidade de conter em si mesma o germe de uma nova sociedade. Essas questões norteavam os debates da Internacional Situacionista na França de 1960. No Brasil, entrementes, esse movimento demorou a aparecer e só ganhou penetração por meio de outros debates. Apenas recentemente o mercado editorial brasileiro editou obras oriundas do movimento. A própria bíblia do situacionismo, a Sociedade do Espetáculo de Guy Debord, ganhou sua versão brasileira apenas em 1997 pela editora Contraponto, trinta anos depois do lançamento francês.
} 
acoplamentos e interpenetrações com os sistemas sociais ${ }^{5}$. Para Luhmann, a comunicação não é nem apenas um sistema nem um ação efetuada por intermédio da linguagem. É uma lógica atávica do sistema.

A comunicação é um elemento central na teoria dos sistemas sociais de Niklas Luhmann. Sistemas sociais, por sua vez, são sistemas de comunicação e a sociedade seria tão somente o mais amplo dos sistemas sociais. O que define um sistema é a separação entre ele mesmo e o ambiente ${ }^{6}$, que como complexidade bruta, seria um exterior não organizado e infinitamente complexo. O interior do sistema, contrariamente, é uma zona de redução de complexidade. Daí a importância da comunicação, pois é por meio dela que se seleciona a informação disponível no ambiente.

Isso quer dizer que a comunicação, tal como entendida por Luhmann, é uma operação exclusiva dos sistemas sociais. Assim, pessoas não comunicam: sistemas sociais comunicam. Pessoas, que na terminologia luhmanniana atendem pelo conceito de sistemas psíquicos, produzem pensamentos, e não comunicação. Comunicação gera mais comunicação, e pensamento gera mais pensamento: tudo separado. Ambos os sistemas são fechados, ou seja, qualquer alteração que venham a sofrer depende exclusivamente das suas próprias operações. É este fechamento operacional que garante autonomia a tais sistemas autopoiéticos, e quando a

5 "Para Niklas Luhmann, a comunicação é alguma coisa muito improvável, muito difícil de acontecer. Pessoas, assim como sistemas sociais, são, em princípio, fechados. Há muita dificuldade em receber informações externas e para que a comunicação tenha algum sucesso, pessoas e sistemas não usam a linguagem, mas acionam 'mecanismos de lubrificação' do processo comunicacional." Marcondes Filho, Ciro. Até que ponto, de fato, nos comunicamos? São Paulo: Paulus, 2004. (p.85).

6 A diferenciação sistema/ambiente seria processada apenas internamente, e de maneira autopoiética. O conceito de autopoiese vem Humberto Maturana, que somado a um mundo de sistemas fechados, produz um cenário onde os sistemas não recebem qualquer input do ambiente. O sistema jamais entra em contato direto com o ambiente e só conhece suas próprias circunstâncias internas. Ainda que o biólogo chileno discordasse de Luhmann dizendo que os seres vivos são autopoiéticos e operacionalmente fechados, mas não os sistemas sociais, o conceito de 'fechamento operacional' permanece como problema epistemológico para a comunicação. Isso porque ao mesmo passo que a dinâmica autopoiética ajuda a entender as esquizofrênicas decisões relacionais da contemporaneidade, também esconde as tênues relações, as fatais interpenetrações e as obscuras lógicas de interação que essa área, a comunicação, não pode se furtar. Ademais, essa categoria entende que toda e qualquer decisão só pode ser feita internamente, o que mina não apenas os conceitos de convencimentos, influência, persuasão, manipulação e outros tão importantes para a área. Mina também o próprio entendimento da comunicação como objeto, como um fenômeno passível de localização e tratamento analítico. 
comunicação acontece, eles não são criadores desta, apenas participam dessa cibernética necessária, desse óleo que viabiliza a sociedade. De certa maneira, a comunicação é a própria sociedade, e o restante são peças desta imensa termodinâmica social.

Ou seja, fora da sociedade não há comunicação. E aquilo que não é comunicação, não faz parte do sistema, sendo então ambiente. Como os seres humanos não fazem parte da sociedade, eles não comunicam, pertencendo assim ao ambiente. Como pessoas (sistemas psíquicos), participamos da sociedade apenas como endereçamentos para a comunicação. O que existe é um acoplamento estrutural entre a sociedade como sistema social e os indivíduos como sistemas psíquicos que, no caso da comunicação, requer a presença destes sistemas acoplados estruturalmente. Mas de novo, esses sistemas não são afetados pelo ambiente que, no máximo, pode estimular operações para o sistema trabalhar internamente.

O diagrama de Luhmann explica com originalidade o movimento dos sistemas sociais, e mesmo a sua corrupção (falência). Mas esse desenho não abarca a complexidade das relações comunicacionais tal como entendem os estudos da comunicação. Para os comunicólogos, a comunicação é necessariamente um acontecimento dado na intersubjetividade do mundo, quer seja produzido lingüisticamente ou não. É uma força social que não se desvincula dos agentes que a produzem, sejam eles denominados emissores, indivíduos, interpretante ou sujeitos. É portanto um entendimento da comunicação contrário a esse entendimento apenas sistêmico, de uma qualidade exclusivamente termodinâmica. No clarividente mundo apresentado por Luhmann, muitas dimensões da comunicação não cabem. Não haveria, por exemplo, a possibilidade de uma comunicação inédita não aceita pelo sistema - pois se não foi aceita, não pode ser comunicação - mas que surte algum efeito.

A teoria luhmanniana da sociedade como comunicação é tanto um achado epistemológico quanto a perdição total. Afinal, como pensar a dinâmica entre sistemas psíquicos sem comunicação? Para Luhmann, os sistemas psíquicos não se comunicam entre si, mas estão apenas acoplados estruturalmente à comunicação, axioma que inviabiliza o conceito de comunicação como mediação e 
compartilhamento. O problema é do todo modo pertinente, pois a pergunta de Hans Ulrich Gumbrecht permanece: é possível não comunicar? Pode-se descomunicar?

O debate de Luhmann com Habermas é famoso, e a proposição do filósofo alemão poderia ser resumida da seguinte maneira: se a linguagem é apenas um meio da comunicação, e se a comunicação e os sistemas têm lógicas diferentes e teorias próprias, o que vai unir estes dois campos de codificação da atividade social? Ainda que aceitemos que a linguagem não é o elemento que provê suficiente complexidade para relacionar individuação humana e socialização, é difícil decalcar apenas a comunicação em toda essa extensão. Ademais, se as estruturas lingüísticas dos sistemas psíquicos e dos sistemas sociais são coisas tão diferentes, então falamos de uma comunicação que, mesmo extensa e operacional, não é o elemento que pode dar unidade à experiência social.

Mas haveria talvez uma outra possibilidade, um outro elemento a unificar a experiência social que não a linguagem, como queria Habermas. É aqui que Hakim Bey, o anarquista americano, pode ajudar. Apesar do programa político de Bey, há no seu famoso ensaio sobre as zonas temporárias elementos que merecem maiores considerações. O mote teórico de Bey trata de estabelecer conexões entre a dinâmica psicológica individual e os sistemas sociais. Apesar de os sistemas sociais serem feitos de muitas consciências (ou sistemas psíquicos, como os entende de maneira homóloga Niklas Luhmann), haveria uma relação de duplo condicionamento nessas instâncias. Ou seja, os sistemas de consciências (psíquicos) estariam umbilicalmente ligados aos sistemas sociais, afetando-se mutuamente. Claro que tal proposição não é nem nova nem estranha à sociologia, que a toma como princípio basilar. Ocorre aqui, entretanto, que Bey vasculha a imbricação entre essas duas instâncias, o plano macro (social) e o micro (individual).

É nessa dificuldade em se trabalhar simultaneamente com macro e micro manifestações sociais que o autor pode contribuir com suas provocações. Esse hiato teórico reflete a insolúvel dificuldade da sociologia em conseguir traçar linhas de sentido entre níveis diferentes, entre temáticas e problemas que ainda que teoricamente conectados, mostram-se operacionalmente auto-excludentes. Isso porque o congelamento da estrutura tem por efeito colateral o extermínio dos 
movimentos, que constitui em si mesmo as conexões entre objetos, o tráfego de sentido entre as diferentes escalas.

Mesmo o conceito de Lebenswelt da fenomenologia husserliana não resolve as correlações entre efeitos da experiência vivida e o consenso da coletividade percebida. Ocorre que esse fundo comum entre indivíduos unidos num contexto dado não coincide com o sistema social que a sociologia fez possível desenhar. A relação não é passível de correlações ou de elucidação mútua. Há então o campo do vivido e uma dimensão social de proporções dantescas, e toda a movimentação entre essas duas escalas repousa silenciosa. Essa pequena mas importante fenda epistemológica permitiu o surgimento de toda uma subárea da sociologia, a comunicação social, cujo princípio seria trabalhar precisamente essa dissensão. O abismo entre as duas escalas é tamanho que o campo do vivido chafurda no plano do inefável, do fluido, do incerto e do mutável7, apartados, por sua vez, por esquemas sólidos mas inertes das manifestações que lhe deram origem: o estável, as estruturas, as instituições, as regulamentações e as leis. O que Hakim Bey pode ajudar-nos a ver é que os modos de passagem entre essas duas instâncias sociais são passíveis de identificação. Seus rastros constituem a natureza do objeto comunicacional.

Haveria nas afecções individuais toda uma visibilidade subestimada, de onde o feixe de ações entre as escalas emergiria, oferecendo-se a quaisquer análises. Seguindo a tradição do americanismo libertário, Bey ilumina a relação de coerção que o sistema social aplica sobre os sistemas psíquicos, agindo como estruturas de controle cujo movimento não é claramente perceptível. O desenho é, por assim dizer, antes o de um atrator estranho que o de um atrator simples, como entende os estudos do caos. Esse princípio de coerção social teria por finalidade limitar a complexidade e

\footnotetext{
7 Não é de agora que o circuito de afecções se faz em espirais fracionadas, não-linerares. Uma reunião ou aglomerado de pessoas não constitui, necessariamente, campo de agenciamento: "Uma vez mais se precisa de um cientista social diferente, capaz de ver dentro da sociedade a existência de manifestações afetivas que não passam mais pelas relações de vizinhança. Podese morar em um prédio de apartamentos, de 20 andares, não conhecer absolutamente nenhum de seus vizinhos nos outros andares e, pelo contrário, embora não os conheça, odiar a todos. É perfeitamente possível. Por outro lado, vai-se a um estádio de futebol em que não se conhece ninguém e todos os que estiverem com a mesma camiseta de seu time, ama-se desesperadamente e, se houver um gol, você beija todos na boca! Como é possível? A afetividade tem outro campo, outro canal de manifestação que não é o mesmo da comunidade. É preciso outra concepção para avaliar a mudança da situação." Sevcenko, Nicolau. O Fim da História. Revista Atrator Estranho Nº 19. São Paulo, NTC, 1996.
} 
diversidade dos padrões de troca nas interações e interpenetrações dos sistemas psíquicos e sociais.

Bey sugere que se remova o sistema de controle não no âmbito social, dado que este não é imediatamente acessível, é sempre construção de um todo com muitas partes, mas no âmbito meramente individual. Sugere, assim, que nos removamos nós mesmos das estruturas de controle, o que teria como efeito a entrada em um espaço onde a dinâmica de relações entre o psíquico e o social seria modulada livremente. É essa concepção de um anarquismo ontológico que propiciaria a formação de ambientes onde a experiência social se faz em espaços livres de autonomia transitória, uma TAZ.

Seriam zonas temporárias ${ }^{8}$. Temporárias porque mesmo que as expressões da pessoalidade se dessem livremente, permaneceria ainda uma influência residual das estruturas de controle no canal de comunicação entre sistema psíquico e social. Ou seja, para cada dilatação do canal comunicativo entre as duas escalas, haveria uma força contrária, espécie de pressão permanente para a dissolução desse canal. Para cada levante expansivo, haveria uma força centrípeta que os submeteria a mecanismos de moderação e organização. Nos termos de Deleuze e Guattari, é a força dos aparelhos de captura.

Apesar da contingência de ser efêmera ${ }^{9}$, a energia de ruptura de uma TAZ é suficientemente grande para superar sua fragilidade temporal. Quando se percebe o que está se passando, quando se toma consciência do processo e se percebe o efeito da TAZ nos espaços de vivência que circulamos, compreendemos toda sua processualidade de uma maneira intuitiva. Isso porque ela não é engendrada pelas

\footnotetext{
8 "A História diz que a revolução conquista 'permanência', ou pelo menos alguma duração, enquanto o levante é 'temporário'. Nesse sentido, um levante é uma 'experiência de pico' se comparada ao padrão 'normal' de consciência e experiência. Como os festivais, os levantes não podem ocorrer todos os dias - ou não seriam 'extraordinários'. Mas tais momentos de intensidade moldam e dão sentido a toda uma vida. O xamã retorna - uma pessoa não pode ficar no telhado para sempre - mas algo mudou, trocas e interrogações ocorreram - foi feita uma diferença”. Bey, Hakim. TAZ: Zona Autônoma Temporária. São Paulo: Conrad, 2001. (p.16).

9 Penso que a saúde das fratrias depende de que elas tenham vida curta. Em outras palavras: o destino de um fratria, cumprida sua função de criadora de linguagem (ou respaldo para experiências com os limites, o que é a mesma coisa), deve ser sua dissolução espontânea, ou no mínimo seu esgarçamento. Kehl, Maria Rita. Existe uma Função Fraterna? IN: Kehl, Maria Rita (org). Função Fraterna. Rio de Janeiro: Relume Dumará, 2000. (p. 45).
} 
ferramentas dos sistemas sociais, é antes efeito de uma abertura dissonante que os sistemas psíquicos imprimem no composto social. A conseqüência imediata é uma compreensão das estruturas de controle e a percepção da uma experiência salutar, sem a mediação deste princípio organizador.

A Babilônia toma suas abstrações como realidade. É precisamente dentro dessa margem de erro que a TAZ surge. Iniciar a TAZ envolve várias táticas de violência e defesa, mas seu grande triunfo está em sua invisibilidade - o Estado não pode reconhecê-la porque a História não a define. Assim que a TAZ é nomeada (representada, mediada), ela deve desaparecer, ela vai desaparecer, deixando para trás um invólucro vazio, e brotará novamente em outro lugar, novamente invisível, porque é indefinível pelos termos do Espetáculo. Assim sendo, a TAZ é uma tática perfeita para uma época em que o Estado é onipresente e todo-poderoso mas, ao mesmo tempo, repleto de rachaduras e fendas.10

Fato é que a dificuldade em se trabalhar com diferentes escalas não é um problema particular da teoria dos sistemas sociais de Niklas Luhmann. É uma invariável nas teorias sociológicas que se manifesta com mais nitidez em Luhmann. É, de toda maneira, uma limitação que o campo da comunicação tem por dever ultrapassar. A natureza do objeto comunicacional, e o obstáculo teórico que a área trata de romper, ficam mais claros quando tomamos um objeto particular, como a internet.

Não constitui objeto específico ao campo da comunicação o medium internet, entendido como canal multifacetado de emissões e recepções. Isto é, o medium como simples instrumento de comunicação. O que constitui objeto são as alterações que esse medium cria, é a ferramenta entendida como condicionante da sociedade e não como produto anódino da evolução tecnológica. Do ponto de vista de Bey, isso quer dizer que a conexão e imersão em ambientes virtuais são experiências

10 Bey, Hakim. TAZ: Zona Autônoma Temporária. São Paulo: Conrad, 2001. (p.18). O paralelo com a filosofia deleuzeana e o ativismo de seu parceiro Felix Guattari é autoevidente: "Não se trata, então, de Universos de referência em geral, mas de domínios de entidades incorporais que se detectam ao mesmo tempo em que são produzidos, e que se encontram todo o tempo presentes, desde o instante em que os produzimos. Eis aí o paradoxo próprio a esses Universos: eles são dados no instante criador, como hecceidade e escapam ao tempo discursivo; são como os focos de eternidade aninhados entre os instantes. Além disso, implicam a consideração não somente dos elementos em situação (familiar, sexual, conflitiva), mas também a projeção de todas as linhas de virtualidade, que se abrem a partir do acontecimento de seu surgimento." Guattari, Félix. Caosmose: Um novo paradigma estético. São Paulo: 34, 1992. (p.29). 
favoráveis à aproximação da máquina social com as movimentações psíquicas. Nesse objeto comunicacional, estende-se não a totalidade de uma sociedade determinada, mas o campo específico de inter-relações entre sistema social e sistema psíquico. $\mathrm{O}$ campo da comunicação.

E foi esse movimento que se pode observar nos primeiros anos da grande rede. O início da internet é rico em registros de indivíduos que procuravam uma TAZ, ou procuravam sua TAZ particular. Da Usenet à darknet, passando por uma miríade de BBS (Mindvox e Well, por exemplo), os anos dourados da grande rede reportam diversas experiências de usuários à procura de uma TAZ. Viajavam então para um lugar distante, onde pudessem se sentir estranhos, em uma língua outra, em uma cultura outra. A desterritorialização é, afinal, um deslocamento que prescinde de mobilidade geográfica. O retrospecto dos últimos vinte anos de internet nos mostra que a TAZ encontrou condições favoráveis nos mundos eletrônicos do TCP/IP11, fermentando os mais variados levantes, dos mais variados espectros ideológicos.

Foram maneiras de viver um poder difuso, maneiras de criar e de destruir por meio de uma estrutura arbórea, descentrada e disjuntiva. É insuficiente olhar essa rede por fora, apoiando-se exclusivamente em estatísticas ou índices sociais, pois o objeto está dentro. O problema da comunicação é esse universo de relações, essa malha ou rede de processos entre o objeto técnico e o usuário.

11 "Como fã do cyberpunk, não consigo deixar de antever o importante papel que o 'hacking da realidade' terá da criação das TAZs. Assim, como Gibson e Sterling, acredito que a net oficial jamais conseguirá conter a web ou a contra-net - a pirataria de dados, as transmissões não-autorizadas e o fluxo livre de informações não podem ser detidos. (Na verdade, no meu entender, a Teoria do Caos pressupõe que nenhum sistema de controle universal seja possível.)”. Bey, Hakim. TAZ: Zona Autônoma Temporária. São Paulo: Conrad, 2001. (p.34). 


\section{REFERÊNCIAS BIBLIOGRÁFICAS}

BEY, Hakim. TAZ: Zona Autônoma Temporária. São Paulo: Conrad, 2001.

DELEUZE, Gilles \& Guattari, Félix. Mil Platôs - Capitalismo e Esquizofrenia. São Paulo: Editora 34, 2000.

GUATTARI, Félix. Caosmose: Um novo paradigma estético. São Paulo: 34, 1992.

GUMBRECHT, Hans Ulrich. Corpo e Forma. Rio de Janeiro, EDUERJ, 1998. Materialities of Communication. Stanford: Stanford University Press, 1994.

HABERMAS, Jürgen. Teoría de la acción comunicativa. Vol. I e II. Madrid: Taurus, 1987. Brasileiro, 1989.

Consciência moral e agir comunicativo. Rio de Janeiro: Tempo

KEHL, Maria Rita (org). Função Fraterna. Rio de Janeiro: Relume Dumará, 2000.

LUHMANN, Niklas. A Improbabilidade da Comunicação. Lisboa: Veja, 1992.

La Sociedad de la Sociedad. México: Herder, 2007.

Soziologische Aufklärung 6. Die Soziologie und der Mensch.

Opladen: Westdeutscher Verlag, 1995.

Introducción a la teoría de sistemas. México: Universidad Iberoamericana, 1996.

Intersubjetividad o Comunicación: Dos Diferentes Puntos de Partida para la Construcción de una Teoría Sociológica. In: Complejidad y Modernidad. De la Unidad a la Diferencia. Madrid: Trotta, 1998.

MARCONDES FILHO, Ciro. Até que ponto, de fato, nos comunicamos? São Paulo: Paulus, 2004.

SEVCENKO, Nicolau. O Fim da História. Revista Atrator Estranho $\mathrm{N}^{0}$ 19. São Paulo, NTC, 1996. 